\title{
Composición quimicomineralógica de las materias primas y ahorro de energía en la industria del cemento
}

FRANCISCO SORIA SANTAMARIA, Dr. en Química Industrial Profesor de Investigación del IETCC/CSIC

\section{RESUMEN}

En el campo del ahorro de energia, principalmente t'érmica, en la industria cementera, no sólo deben contemplarse las posibilidades en cuanto al proceso tecnológico o el producto acabado, sino también la influencia de la composición quimicomineralógica de las materias primas. Se analizan los distintos parámetros que inciden en la reactividad de los crudos, tales como tamaño de grana (finura), composición quimica y mineralógia de los componentes principales.

A continuación se hace una exposición de la importancia de los componentes minoritarios como fundentes y mineralizadores, destacando finalmente el interés y comportamiento práctico de alguno de ellos.

\author{
$S U M M A R Y$
}

In the field of energy saving, mainly thermic, in the cement industry, not only should we consider the possibilities in the technological process or the finished product, but also the influence of the chemical-mineral composition of the raw materials.

The distinct parameters which fall into the reactivity of the crudes are analyzed, such as the size of the grain (fineness), chemical and mineral composition of the principal components. There follows an exposition of the importance of the minority components as smelting and mineralizers, finally emphasizing the interest and practical behaviour of some of them.

\section{INTRODUCCION}

La industria del cemento se incluye en la denominación de industria pesada, tanto por su volumen de inversión y producción como por el elevado consumo energético en la fabricación.

España ocupa el sexto puesto en el mundo con una producción prevista para este año 1982 próxima a los 30 millones de toneladas, un consumo térmico de unos 3,2 millones de $t$ de carbón ( $6.500 \mathrm{kcal} / \mathrm{kg}$ clínker) y 0,6 millones de $\mathrm{t}$ de fueloil y un consumo de energía eléctrica de unos 3.000 millones de $\mathrm{kW} \cdot \mathrm{h}$.

La energía térmica es la más importante, siendo del orden de 8 a 10 veces superior a la energía eléctrica.

Por ello, gran parte del esfuerzo se ha centrado en el ahorro de energía térmica, enfocando el problema hasta ahora en dos aspectos: el proceso tecnológico y el producto acabado.

El proceso tecnológico ha considerado de modo permanente el ahorro energético, logrando notables mejoras desde la introducción de los intercambiadores de calor en vía seca (suspensión gaseosa) o semiseca (parrilla), con la reducción gradual del proceso por vía húmeda. La inclusión de la precalcinación en la década de los 70 , reduciendo el volumen de horno a igualdad de producción, con ligeras mejoras en el consumo específico, supuso 
un nuevo perfeccionamiento. Quedan, quizás, por considerar nuevas modificaciones para mejorar el proceso que tiendan a acelerar las reacciones en el intervalo muerto entre $1.000-1.300^{\circ} \mathrm{C}$, en el que se crean sólidos nacientes antes de la aparición de la fase líquida con peligro de su "envejecimiento" y pérdida de reactividad.

El empleo de forma creciente de componentes secundarios o adiciones incorporadas al clínker en la fase final de molienda de cemento es, quizás, la forma más eficaz e inmediata de ahorrar energía y, simultáneamente, crear nuevos cementos con propiedades adicionales sobre las del propio cemento portland-base.

Existe una tercera vía de participación con nuevas mejoras en el ahorro energético que la constituyen las materias primas, contempladas desde su origen, naturaleza, composición químico-mineralógica, dosificación, empleo de correctores, etc.

Este es el tema que se va a tratar en esta exposición.

\section{MATERIAS PRIMAS Y CONSTITUCION DE CRUDOS}

Desde los primeros tiempos en la historia del cemento portland se centró la atención en las causas de su hidraulicidad, lo que condujo a considerar, como factor prioritario, la composición química de las materias primas que entran en su composición.

Surge la era de los índices, los módulos y los factores de saturación, basados en la formación en el producto final de unos minerales, a veces hipotéticos, pero que servían para dirigir o regular la calidad del producto final.

En todos los casos, se consideraba el contenido de los cuatro óxidos principales $\left(\mathrm{SiO}_{2}\right.$, $\mathrm{Al}_{2} \mathrm{O}_{3}, \mathrm{Fe}_{2} \mathrm{O}_{3}, \mathrm{CaO}$ ) y se establecían límites, a veces muy estrechos, para conseguir, en condiciones técnicas aceptables, ese producto que quedaba definido como cemento portland.

El resto de los componentes, llamados menores o secundarios, se procuraba mantenerlos bajos conscientemente $\left(\mathrm{MgO}, \mathrm{SO}_{3}\right.$, álcalis, etc.), bien por las dificultades que pudieran provocar en el proceso de fabricación o bien por considerarse nocivos en el comportamiento del cemento.

La posible capacidad de cocción se valoraba mediante ensayos empíricos, empleando como guía el grado de combinación de la cal (componente básico prácticamente único) en unas condiciones preestablecidas de tratamiento. De ello se deducía una norma general para la selección de materias primas, según la cual, unos crudos con alto contenido en sílice libre, bajo contenido en fundentes y módulos elevados de silicatos y fundentes, sinterizan a temperaturas altas (duros de cocer) en comparación con crudos que tengan bajos los valores antes citados (blandos de cocer).

Esta ha sido, durante muchos años, la única regla que daba oportunidad para un ahorro de combustible en la industria del cemento.

Pero la experiencia ha demostrado que las pequeñas variaciones en la composición quuímica de los crudos no bastan para explicar su comportamiento, a veces notablemente distinto en el curso de la clínkerización y, por tanto, para prever, en el caso de una nueva instalación, la aptitud a la clinkeriación del crudo elegido.

Entre los parámetros que intervienen en la facilidad de cocción, aparte de la composición química, pueden citarse: 
- Finura de molienda.

- Homogeneidad química y homogeneidad mineralógica.

- Mineralogía de las materias primas y grado de cristalinidad.

- Presencia de componentes menores y su influencia en las reacciones de clinkerización.

- Proporción y composición de las fases líquidas que puedan surgir en el curso de la cocción.

Medir cuantitativamente cada uno de estos factores es difícil, y lo que se ha tratado siempre es establecer, mediante ensayos de laboratorio, la aptitud a la clinkerización de los crudos como medida global cle la influencia de dichos factores.

\subsection{Aptitud a la cocción. Reactividad}

Dos son los parámetros que dan cuenta de la forma que el crudo se transforma en clínker: la aptitud a la cocción y la reactividad.

Puesto que los constituyentes principales del clínker son todos cornpuestos de calcio, la transformación vendrá caracterizada, en principio, por el grado de combinación de la cal en los compuestos formados, por el porcentaje de cal libre, por la temperatura y por el tiempo.

De los valores de los dos primeros, en función de los dos últimos (a constancia de uno de ellos) se cuantifica la aptitud a la cocción del crudo.

La reactividad, por otra parte, es un concepto dinámico que se define por la velocidad de una reacción o conjunto de reacciones a una temperatura dada.

La aptitud a la cocción depende de la saturación en cal y de la reactividad de las materias primas. Esta, a su vez, es función del módulos de silicatos, del módulo de fundentes, del tamaño de grano, de su naturaleza mineralógica y de su estado de actividad en el momento de entrar en combinación.

Esto último es de capital importancia y explica la diferencia de reactividad de un mismo material cuando se encuentra en crudos diferentes. La explicación de estos efectos se funda en las leyes de la difusión de sólidos, singularmente en las relaciones entre los coeficientes de difusión sólida y la energía de activación del material, la cual depende en sí de los defectos estructurales de los sólidos en presencia. Otros parámetros, singularmente los de la fase líquida como viscosidad, mobilidad iónica y tensión superficial juegan igualmente un papel esencial.

Para un crudo dado, tal como queda definida, la reactividad hace abstracción de todo carácter de calidad del clínker obtenido y sí se relaciona con las necesidades energéticas del proceso y con la capacidad de la instalación que lo lleva a cabo.

\subsection{Métodos para medir la aptitud a la coción}

Los métodos existentes para medir la aptitud a la cocción, tanto teóricos como experimentales, son numerosos.

Entre los métodos experimentales se pueden citar los siguientes:

a) Medida del residuo insoluble en $\mathrm{ClH}$ 1/10 de un crudo después de permanecer un 
cierto tiempo a $1.000-1.050^{\circ} \mathrm{C}$ (Método de Hendrick). Valores por debajo de $8 \%$ se califican de muy buenos, buenos o medios entre 8 y $12 \%$ y mediocres o malos por encima de $12 \%$. Este método es antiguo y, por su baja temperatura de ensayo, deja de considerar la zona más importante del proceso en que aparece la fase líquida.

b) Medila de la cal libre por tratamiento a una temperatura dada durante un tiempo definido. Son los métodos más numerosos con temperaturas que oscilan de 1.300 a $1.450^{\circ} \mathrm{C}$ y tiempos comprendidos entre 15 y 45 minutos (1) (2) (3). Naturalmente, valores crecientes del contenido en $\mathrm{CaO}$ libre, indican reactividades decrecientes.

c) Medida del tiempo necesario para que la cal libre sea igual o inferior a un valor preestablecido, para una temperatura dada de tratamiento. Por ejemplo: Tiempo para que $\mathrm{CaO} \leqslant 2 \%$ a $1.350^{\circ} \mathrm{C}(4)$.

d) Medida de la relación entre la $\mathrm{CaO}$ total y el máximo de $\mathrm{CaO}$ libre en el intervalo $700^{\circ} \mathrm{C} \leqslant \mathrm{t} \leqslant 1.400^{\circ} \mathrm{C}(5)$.

Esta definición se basa en la hipótesis de que la cantidad máxima de $\mathrm{CaO}$ libre durante el proceso es tanto menor cuanto más reactivo es el crudo. Es siempre mayor de 1 el factor y aumenta cuando la reactividad crece: $\mathrm{K}=\mathrm{CaO}$ t/CaO máx.

e) Medida del factor de "aptitud a la cocción" AC (6), como expresión cuantitativa del desarrollo de la $\mathrm{CaO}$ libre, en el intervalo de $1.000-1.450^{\circ} \mathrm{C}$.

Para este ensayo se preparan 7 muestras, compactadas en condiciones preestablecidas, y se introducen en un horno previamente calentado a $700^{\circ} \mathrm{C}$. Al llegar a $1.000^{\circ} \mathrm{C}$ se retira la primera muestra, $\mathrm{C}_{0}$, al cabo de 20 minutos. Acabado este tiempo, se eleva lo más rápidamente posible la temperatura a $1.100^{\circ} \mathrm{C}$ y se mantiene otros 20 minutos antes de retirar la segunda muestra, $\mathrm{C}_{1}$; se eleva a $1.200^{\circ} \mathrm{C}, 20$ minutos más, $3 .^{\mathrm{a}}$ muestra, y así, sucesivamente a $1.300,1.350,1.400$ y $1.4500^{\circ} \mathrm{C}$ (figura 1 ).

Como resultado, en vez de la integral de la curva cal libre en función de la temperatura, se toma la relación de dos superficies: una de referencia ( $50 \%$ de $\mathrm{CaO}$ libre$1.450^{\circ} \mathrm{C}$ ) y otra la delimitada por la curva real de la $\mathrm{CaO}$ libre en función de la temperatura. Esto conduce a la expresión:

$$
A C=\frac{600}{C_{0}+2 C_{1}+2 C_{2}+3 C_{3}+4 C_{4}+4 C_{5}+2 C_{6}}
$$

De los métodos hasta aquí citados, el último tiene la ventaja de considerar el desarrollo completo de la combinación de $\mathrm{CaO}$ en presencia de líquido, el desarrollo de las reacciones en estado sólido e, incluso, el final de la descarbonatación (valor $C_{0}$ ).

f) Un método más rápido y más fiable que los anteriores, que obtienen información valorando el contenido de cal libre de muestras calcinadas en serie, se basa en la observación continua por difracción de rayos $\mathrm{X}$ de las distintas fases que se forman o desaparecen en un tratamiento térmico previamente programado (7).

Este método permite observar las diferencias de la clinkerización debidas a la composición mineralógica del crudo y deducir la efectividad de mineralizadores, haciendo una valoración de su aptitud (figura 2).

La curva 1 de la figura es característica para los casos en que hay un mineralizador efectivo en el crudo. Su efecto se manifiesta en la aparición del primer pico y en la pendiente posterior de la curva. 


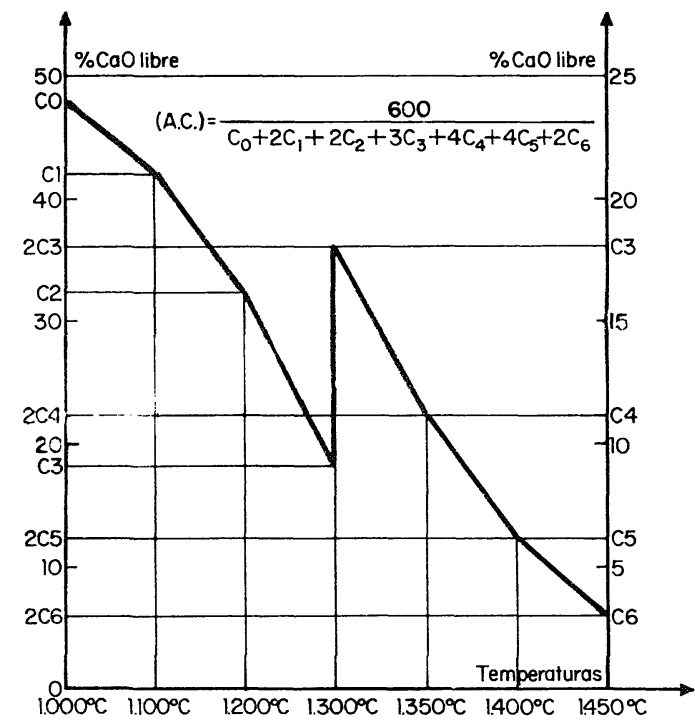

Aptitud a la cocción según BLAISE, MUSIKAS Y TIEDRED.

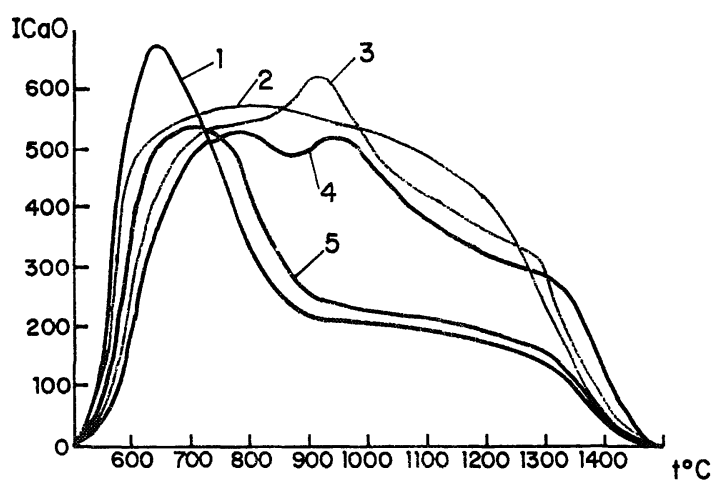

Fig. 2

Fig. 1

La curva 2 indica alta velocidad de disociación del $\mathrm{CaCO}_{3}$ y curso retardado del proceso mineralizador, lo que muestra la baja capacidad de reacción del crudo.

Las curvas 3 y 4 son típicas de crudos con baja capacidad de reacción del componente carbonatado (marmol o caliza dura). El pico máximo se retrasa hasta $900^{\circ} \mathrm{C}$ y se perturba la combinación de esta cal libre en los minerales del clínker.

La curva 5 se relaciona con los casos en que el crudo contiene óxidos tales como $\mathrm{R}_{2} \mathrm{O}$, $\mathrm{SO}_{3}$ y $\mathrm{MgO}$, destacando su efecto mineralizante.

Es de resaltar, una vez más, que estas medidas, por precisas que sean, dan resultados cuyo significado no deja de ser relativo ya que el proceso tecnológico se desarrolla en condiciones muy distintas a las del laboratorio y diferentes entre sí, según el tipo de instalación en que se llevan a cabo.

Hay otros métodos que calculan la aptitud a la cocción a partir de parámetros químicos, físicos y mineralógicos.

a') Así, uno de ellos (4) muestra que la aptitud a la cocción "experimental o práctica" (tiempo de reacción a $1.350^{\circ} \mathrm{C}$ necesario para reducir la cal libre a menos de $2 \%$ ) coincide con la aptitud a la cocción teórica calculada por una ecuación donde se incluye: el residuo en 90 micras (con lavado previo de finos aglomerados) $\mathrm{H}_{\mathrm{R} 90}$, el contenido en fase líquida a $1.350^{\circ} \mathrm{C}$, según $\mathrm{Dahl}$ (del crudo desprovisto de pérdida por calcinación) SA y el standard de cal, según Spohn y colaboradores $K_{\mathrm{ST}}{ }^{\mathrm{III}}$ (8):

$$
B_{\mathrm{t}}=55,5+11,9 H_{\mathrm{R} 90}+1,56\left(K_{\mathrm{ST}_{\mathrm{T}}}^{\mathrm{III}}-90\right)^{2}-0,43(\mathrm{SA})^{2}
$$

b') Christensen (9), señala que es posible calcular la cal libre a $1.400^{\circ} \mathrm{C}$, con un tratamiento de 30 minutos, en función de la composición química y del contenido de partículas de calcita $>125$ micras y de cuarzo $>44$ micras. Esto concuerda con lo dicho en el método anterior.

c') Finalmente, Fundal (10) presenta una expresión según la cual el contenido de cal 
libre a $1.400^{\circ} \mathrm{C}$ durante 30 minutos de cocción viene dada por:

$$
X=0,33 L S F+0,018 M S+0,56 K+0,93 Q-0,349
$$

donde:

$L S F=$ Standar de cal, según Kühl.

$M S$ = Módulo silícico.

$\mathrm{K}=$ Porcentaje de $\mathrm{CaCO}_{3}>125$ micras.

$Q \quad=$ Pocentaje de cuarzo $>44$ micras.

Todos estos métodos teóricos o experimentales han sido desarollados, tanto por constructores de maquinaria, como por fabricantes de cemento.

El constructor de maquinaria desea, para una mezcla dada, preconizar una finura de molienda para conseguir un buen clínker en condiciones "normales" de cocción.

Por otro lado, al fabricante de cemento, los ensayos de aptitud le permiten optimizar la composición del crudo (con adiciones eventuales, por ejemplo) o la finura de molienda, y seguir el comportamiento del crudo en el horno por observación de la curva de cal libre en función de la temperatura.

En cualquier caso, para un crudo dado, lo que se trata es de conservar la molienda al mínimo compatible con una buena cocción, a fin de reducir el consumo de energía de la fábrica proyectada (fabricante de maquinaria) o ya en marcha (fabricante de cemento). $\mathrm{Pa}-$ ra que el ensayo se aproveche adecuadamente, es preciso que en la molienda de laboratorio se obtengan curvas granulométricas representativas del molino industrial.

Vistos globalmente los conceptos de aptitud a la cocción y reactividad, se analizará a continuación la participación en dichos conceptos de los parámetros más importantes ya citados: dimensión de partículas, composición química principal, composición mineralógica y participación de componentes secundarios o minoritarios.

\section{TAMAÑO DE GRANO Y REACTIVIDAD}

Está demostrado por varios autores (11) (12) que el tiempo de disociación del carbonato cálcico $\mathrm{CaCO}_{3}$ es función lineal del diámetro de las particulas, en el entorno de las dimensiones usuales en un crudo de cemento.

Por otra parte, el tiempo necesario para la disociación isoterma de la unidad de masa del mineral arcilloso (distribuida en partículas de intervalo granulométrico muy estrecho) es, aproximadamente, proporcional a la inversa de la superficie específica de dichas partículas:

$$
M^{\prime}=\frac{K^{\prime}}{S_{M}^{\prime}}, \mathrm{sg}^{-1}
$$

Algo similar, y en condiciones análogas, ocurre con las partículas de $\mathrm{CaCO}_{3}$ (variando la constante $K^{\prime}$ ) en la fase del proceso en que la velocidad de disociación es más elevada que la de difusión del $\mathrm{CaO}$ en los óxidos de carácter ácido.

En los procesos simples de difusión binaria entre los óxidos fundamentales, la dirección 
de transporte respeta la regla de De Keyser (13), según la cual, los óxidos con menor afinidad por el oxígeno se difunden en los óxidos con mayor afinidad, es decir,

$$
\begin{aligned}
& \mathrm{CaO}(0,70)-\mathrm{SiO}_{2} \quad(2,39) \\
& \mathrm{CaO}(0,70)-\mathrm{Al}_{2} \mathrm{O}_{3}(1,66) \\
& \mathrm{Al}_{2} \mathrm{O}_{3}(1,66)-\mathrm{SiO}_{2} \quad(2,39)
\end{aligned}
$$

(entre paréntesis se encuentran los valores de afinidad por el oxígeno, según Katelaar).

De acuerdo con esta regla, en la difusión en fase sólida, la reactividad vendrá controlada principalmente por el tamaño de grano de los óxidos receptores de cal, es decir por los óxidos de carácter ácido $\mathrm{SiO}_{2}, \mathrm{Al}_{2} \mathrm{O}_{3}$ y $\mathrm{Fe}_{2} \mathrm{O}_{3}$, siendo el tiempo exigido para la reacción isoterma de la unidad de masa del óxido receptor aproximadamente proporcional a la inversa del cuadrado de su superficie específica (se entiende, en teoría, que las partículas se componen de un solo grano cristalino de una única especie mineralógica).

Finalmente, la clinkerización viene controlada por el proceso de difusión en fase líquida (14). La velocidad de disolución del $\mathrm{C}_{2} \mathrm{~S}$ y $\mathrm{CaO}$ en esta fase líquida depende de la dimensión de sus granos y del "poder mojante" de la fase líquida.

El modelo descrito para el proceso, desde la disociación de minerales arcillosos y descarbonatación del $\mathrm{CaCO}_{3}$, pasando por las reacciones en fase sólida y concluyendo en la clinkerización, permiten deducir algunas conclusiones prácticas.

El diámetro de partícula de los óxidos de carácter ácido (que figura en $4 .^{a}$ potencia) tendrá un efecto decisivo sobre la cinética de las reacciones, tanto en fase sólida como en presencia de líquido. Esto explica, por ejemplo, la gran importancia, para la reactividad de un crudo, de las dimensiones del cuarzo que contiene y de la menor influencia en las reacciones en fase sólida del tamaño de grano de la calcita (que figura sólo al cuadrado).

Los granos más gruesos de fase única presentes en un crudo industrial son siempre los últimos en completar las reacciones. Por eso, a igualdad de superficie específica, la reactividad se acentúa para un huso granulométrico estrecho. En este sentido se debe reconocer que las técnicas empleadas en la práctica para la molienda de crudo no son las más idóneas para lograr una granulometría más reactiva con el menor consumo de energía en el horno (poca precisión de los separadores y molienda conjunta de productos de distinta dureza).

\section{COMPOSICION QUIMICA DEL CLINKER}

La composición química del clínker, expresada en óxidos, viene definida por los valores de uno o varios parámetros característicos, convenientemente elegidos sobre la base de la composición de las materias primas disponibles (tabla I).

La fórmula de Guttman y Gille, que contempla la formación en el grado de máxima saturación de $\mathrm{C}_{3} \mathrm{~S}, \mathrm{C}_{3} \mathrm{~A}$ y $\mathrm{C}_{4} \mathrm{AF}$, sólo se puede cumplir con un enfriamiento en condiciones de equilibrio, inalcanzable en la práctica. Lo mismo ocurre al considerar la composición potencial, de acuerdo con las fórmulas de Bogue.

Según Spohn, el clínker no se enfría en condiciones de equilibro y presupone la formación de $\mathrm{C}_{3} \mathrm{~S}, \mathrm{C}_{2} \mathrm{~A}$ y $\mathrm{C}_{2} \mathrm{~F}$. La relación $C / S P$ lo definió Kühl como standard de cal $\left(K_{\mathrm{ST}_{\mathrm{T}}}{ }^{\mathrm{I}}\right)$. Lea y Parker tratan también de la cantidad máxima de cal combinable en el clínker no 
enfriado en condiciones de equilibrio. La relación $C / L P$ se llama factor de saturación en cal $(L S F)$ o, incluso, standard de cal modificado $\left(K_{\mathrm{ST}}^{\mathrm{II}}\right)$.

Spohn (8) modifica su fórmula e incluye la posible combinación de hasta $2 \%$ de la magnesia en el clínker, liberando, en principio, $0,75 \%$ en peso de $\mathrm{CaO}$ por cada $1 \%$ de $\mathrm{MgO}$. Valores superiores a $2 \%$ de $\mathrm{MgO}$ no afectan la combinación de $\mathrm{CaO}$.

TABLA I

Parámetros de la composición del clínker

\begin{tabular}{|c|c|c|}
\hline Símbolo & Parámetro & Denominación \\
\hline GG & $2,8 S+1,65 A+0,35 F$ & $\begin{array}{l}\text { CaO máx. combinable según Guttman y } \\
\text { Gille }\end{array}$ \\
\hline SP & $2,8 S+1,1 A+0,70 F$ & CaO máx. combinable según Spohn \\
\hline $\mathbf{L P}$ & $2,8 S+1,18 A+0,65 F$ & CaO máx. combinable según Lea y Parker \\
\hline BO & $\%$ de $\mathbf{C}_{3} \mathbf{S}, \mathbf{C}_{2} \mathbf{S}, \mathbf{C}_{3} \mathbf{A},\left(\mathbf{C}_{2} \mathbf{F}\right)$ y $\mathbf{C}_{4} \mathbf{A F}$ & "Composición potencial" según Bogue \\
\hline MS & $S:(A+F)$ & Módulo silícico \\
\hline MF & $A: F$ & Módulo de alúmina o de fundentes \\
\hline MH & $C: S+A+F$ & Módulo hidráulico \\
\hline $\mathbf{M S O}_{3}$ & $\bar{S}: 0,85 \bar{K}$ & Móclulo de sulfatos \\
\hline
\end{tabular}

OBSERVACION: Los símbolos $S, A, F, C, \bar{S}$ y $\bar{K}$ representan los tantos por cientos en peso, respectivamente, de $\mathrm{SiO}_{2}, \mathrm{Al}_{2} \mathrm{O}_{3}, \mathrm{Fe}_{2} \mathrm{O}_{3}, \mathrm{CaO}, \mathrm{SO}_{3}$ y $\mathrm{K}_{2} \mathrm{O}+\mathrm{Na}_{2} \mathrm{O}$ equiv. en el clínker.

Así crea un nuevo standard de cal modificado $\left(\mathrm{K}_{\mathrm{ST}}^{\mathrm{III}}\right)$ como relación $(\mathrm{CaO}-0,75 \mathrm{MgO}) /$ $/ L P$ para $\mathrm{MgO} \leqslant 2 \%$ y $(\mathrm{CaO}-1,5 \mathrm{MgO}) / L P$ para $\mathrm{MgO} \geqslant 2 \%$.

Christensen (15) ha llegado a una expresión semejante para el LSF corregido para el $\mathrm{MgO}(<2 \%)$ sobre la base de las relaciones de fase en el sistema C-A-S-F-M:

$$
L S F_{\mathrm{I}}=\frac{\mathrm{CaO}-0,77 \mathrm{MgO}}{2,8 S+1,18 A+0,66 F}
$$

\subsection{Composición química y reactividad}

Al aumentar la saturación en cal de un crudo, aumenta el número de partículas de $\mathrm{CaCO}_{3}$ "coordinadas" por cada particula de óxidos de naturaleza ácida; por tanto, la fracción de $\mathrm{CaO}$ (sobre la masa total presente) que se difunde en esta partícula ácida, por unidad de tiempo, disminuye. La aptitud a la cocción disminuye.

$\mathrm{Al}$ aparecer la fase líquida $\left(>1.300^{\circ} \mathrm{C}\right.$ aproximadamente) la cinética del proceso de disolución se afecta poco por el contenido en cal presente (saturación), a igualdad de todo lo demás.

Los procesos que tienen lugar en presencia de fase líquida se influencian por la cantidad, composición y propiedades de ésta.

En el sistema cuaternario $C-S-A-F$, aparece a $1.338^{\circ} \mathrm{C}\left(1.301^{\circ} \mathrm{C}\right.$ en saturación de $\left.\mathrm{MgO}\right)$ para $A / F=1,23$ (1,63 en saturación de $\mathrm{MgO}$ ). Es decir, $\mathrm{Al}_{2} \mathrm{O}_{3}$ y $\mathrm{Fe}_{2} \mathrm{O}_{3}$ actúan como fundentes 
de primera fusión, respectivamente, para crudos con $A / F<1,23(<1,63)$ ó $A / F>1,23$ $(>1,63)$.

A igualdad de módulo silícico, la cantidad de fase líquida es máxima para $A / F=1,23$ $(1,63)$; a igualdad de módulo de fundentes la cantidad de fase líquida aumenta al disminuir el módulo silícico.

La viscosidad de la fase líquida disminuye, a temperatura constante, cuando $A / F$ disminuye, teniendo como consecuencia un aumento en la velocidad del proceso de disolución.

Una vez que los aluminatos y ferritos han pasado a la fase líquida, la viscosidad depende esencialmente del módulo de silicatos y de la saturación en cal en dicha fase, aumentando al elevarse ambos parámetros y reduciéndose, por tanto, la reactividad.

Sin embargo, las consideraciones expuestas sobre la influencia de las variaciones del módulo de fundentes no concuerdan claramente con los resultados experimentales y, aunque los crudos con módulo de fundentes comprendido entre 1,3 y 1,6 se juzgan universalmente como los más fáciles de cocer, a igualdad de todo lo demás, hay casos en que esta circunstancia se da con valores del módulo, incluso inferiores a 1 (16).

\section{MINERALOGIA DE LAS MATERIAS PRIMAS}

La aptitud a la cocción de los crudos para la fabricación de cemento se relaciona directamente con la textura de las rocas que lo integran, con su estructura (es decir, con las especies mineralógicas presentes) y con su estado de actividad (grado de cristalinidad), con más fuerza, quizás, que con los parámetros que usualmente se manejan en la práctica, ya citados, como son la composición química, la finura de molienda o el grado de homogeneización.

Tanto en las reacciones en estado sólido como las que tienen lugar en presencia de la fase líquida, la energía de activación, en todas sus fases, es una medida de la rigidez química del edificio reticular.

Cada especie mineralógica, que aporta uno o varios óxidos al crudo, lleva consigo un valor de la energía de activación característico de la especie.

Este valor, dentro de una especie mineralógica, es función del estado de actividad particular en que se encuentra por su formación y vicisitudes derivadas con el tiempo. Imperfecciones en la red, estructuras defectuosas y desórdenes de cualquier otro tipo, aumentan la reactividad. Las soluciones sólidas, del tipo que sean, proporcionan mayores niveles de energía libre.

Del mismo modo, los pseudosólidos y materiales mal cristalizados, o en estado de cristalización incipiente (vidrios, coloides, etc.) deben su gran reactividad al elevado contenido de energía libre de sus estructuras. Por el contrario, el "envejecimiento" de las estructuras aumenta su estabilidad y la dimensión de sus granos y, en consecuencia, los hace menos reactivos.

\subsection{Minerales portadores de óxidos de carácter ácido}

Los minerales principales de arcillas, pizarras, esquistos, etc., son portadores de óxidos de carácter ácido.

Con el mismo fin, se emplean, con menos frecuencia, rocas volcánicas a base de vidrios 
metaestables como tobas, puzolanas, basaltos, pómez etc., o subproductos industriales como cenizas volantes, escorias, lodos residuales (nefelina), etc.

Si es preciso, pueden incorporarse como correctores de naturalea ácida, pequeñas cantidades de materiales silíceos, bauxita, limonita, siderita o cenizas de pirita tostada, según el óxido que se encuentre en defecto y las disponibilidades del mercado.

Entre los óxidos de carácter ácido se encuentra en primer lugar la sílice, que presenta una reactividad variable, atendiendo a su origen, grado de cristalinidad, dimensión de grano, etc.

Según Makashev (17), la reactividad de los distintos tipos de sílice, libre y combinada, aumenta en el orden:

cuarzo $<$ calcedonia $<$ ópalo $<\alpha$-cristobalita $<\alpha$-tridimita $<$ sílice de feldespatos (ortosa; albita; anortita) $<$ sílice de micas y anfiboles $<$ sílice de los minerales de la arcilla $<$ sílice de vidrios de escoria $<$ sílice de vidrios naturales de origen volcánico.

Para alúmina y óxido de hierro, los otros dos óxidos de carácter ácido considerados principales, pueden hacerse consideraciones similares a las de la sílice.

Los sílicatos más abundantes en la fracción arcillosa del crudo son, en general, la illita, las cloritas y la moscovita, siendo la caolinita frecuente pero menos abundante.

La gibsita, boehmita y otros minerales de aluminio son más bien constituyentes esenciales de sedimentos residuales (bauxitas, lateritas, limonitas, etc.), aunque por su pobre cristalinidad presentan un grado elevado de actividad.

Los portadores más comunes de $\mathrm{Fe}^{3+}$ son la glauconita (illita férrica) y la nontronita (montmorillonita férrica). Por su estado peculiar de actividad, las cenizas de pirita tostada son los correctores portadores de hierro más empleados.

La estabilidad de la red de los silicatos viene definida por las temperaturas de principio y fin de la pérdida de agua combinada, que son tanto más bajas cuanto menor es la simetría interlaminar. El orden de estabilidad, según algunos autores (18) (19), es el siguiente:

$$
\text { moscovita }>\text { montmorillonita }>\text { clorita }>\text { illita }>\text { caolinita. }
$$

Aunque estos autores relacionan este orden de estabilidad con la reactividad frente al carbonato cálcico, otros (20) (21) encuentran más correcto relacionar esta actividad, no con la actividad de la estructura antes de su destrucción considerada aisladamente, sino más bien con la temperatura a la cual se crean los óxidos ácidos nacientes en relación con la temperatura a la cual, la disociación del $\mathrm{CaCO}_{3}$ en su presencia, alcanza valores apreciables. Es decir, que también hay que tener en cuenta la naturaleza y textura de la roca carbonatada con la cual se ponen en presencia.

De todas formas, teniendo en cuenta las exigencias de composición química de los crudos industriales, cuando en las arcillas-base la relación $S / A$ es baja (caso de los silicatos más reactivos), van siempre acompañadas en el crudo con valores altos de sílice libre (o combinada en otros silicatos) poco reactivos.

\subsection{Minerales portadores de cal}

La calcita y, rara vez y en pequeñas proporciones, el aragonito y la ankerita, es el principal portador de $\mathrm{CaO}$ en el crudo. 
La reactividad del $\mathrm{CaO}$ resultante de la descomposición del $\mathrm{CaCO}_{3}$ frente a los óxidos ácidos, viene dada por la dimensión de los cristales de calcita, por posibles defectos de red (variación de la temperatura de formación de $\mathrm{CaO}$ ) y por el tiempo en que el $\mathrm{CaO}$ naciente producido empieza a "envejecer" antes de entrar en reacción con otras fases sólidas.

En sedimentos carbonatados como las margas, se ha demostrado que las dimensiones de grano son inversamente proporcionales al contenido de minerales arcillosos. Esto explica la gran facilidad de cocción de estos materiales y la preferencia que los cementeros dan a las calizas de estructura "contaminada" frente a las rocas carbonatadas puras. Los peores carbonatos para el cemento, en lo que se refiere al consumo energético, son las calizas puras y de grandes cristales como, por ejemplo, los mármoles.

\section{COMPONENTES SECUNDARIOS O MENORES}

Bajo este concepto se engloban las distintas especies químicas, distintas de los cuatro óxidos principales, que participan en el proceso de fabricación del clínker.

Estos componentes surgen como minerales accesorios de las rocas, como aportación de los combustibles (cenizas o productos volátiles procedentes de los carbones), de los refractarios, o del desgaste de elementos metálicos, principalmente del proceso de molienda (22). Circunstancialmente, surgen como adiciones incorporadas al crudo con fines específicos.

Los estudios estadísticos llevados a cabo por diversas Sociedades cementeras europeas, sobre unos 150 crudos, muestran que los valores medios de concentración, entre los componentes menores más importantes, disminuyen en el siguiente orden:

$$
\mathrm{MgO}>\mathrm{K}_{2} \mathrm{O}>\mathrm{SO}_{3}>\mathrm{Na}_{2} \mathrm{O}>\mathrm{TiO}_{2}>\mathrm{Mn}_{2} \mathrm{O}_{3}>\mathrm{P}_{2} \mathrm{O}_{5}>\mathrm{SrO}>\mathrm{F}^{1-}>\mathrm{Cl}^{1-}>\mathrm{Cr}_{2} \mathrm{O}_{3}
$$

Los componentes menores se han considerado tradicionalmente como impurezas, contemplando solamente su trascendencia en las propiedades del cemento final. Por eso, a algunos de ellos afectan principalmente las limitaciones de composición en las Normas o Pliegos de Condiciones.

Hoy día, sin embargo, ha adquirido interés primordial el estudio de la presencia de componentes minoritarios e, incluso, de su incorporación como adiciones, con varios fines:

- obtener crudos más reactivos;

- aumentar la eficacia y economía del proceso;

- ampliar la gamma de yacimientos de materias primas con mayor nivel de impurezas (incluyendo desechos industriales) y de combustibles utilizables;

- producir cementos de más calidad.

En el proceso de cocción, estos componentes menores ejercen tres acciones principales:

- modificar las propiedades de la fase líquida;

- trastocar el equilibrio de fases;

- modificar la estructura de los minerales del clínker.

Unos se llaman fundentes, que son sustancias que reducen la temperatura de formación de la fase líquida. Otros, en muchos casos los mismos, se llaman mineralizadores que son compuestos que, por el procedimiento que sea, aceleran la velocidad de un proceso o de una reacción, tanto en fase sólida, en fase líquida o en la interfase sólido-líquido (22). 
La presencia de fundentes es importante, por ser necesaria la fase líquida en la formación del $\mathrm{C}_{3} \mathrm{~S}$, por evitar el "envejecimiento" del $\mathrm{CaO}$ y $\mathrm{C}_{2} \mathrm{~S}$ (sólidos) destinados a disolverse en dicha fase y, por último, por ahorrar energía en el proceso, ya que este ahorro es tanto más acusado cuanto más baja es la temperatura de aparición de la fase líquida.

Basta recordar que en el sistema cuaternario $C-S-A-F$, la fase líquida aparece a $1.338^{\circ} \mathrm{C}$, según Lea y Parker. La presencia de $5 \%$ de MgO, como ya se ha dicho, la reduce a $1.301^{\circ} \mathrm{C}$. Si consideramos el $\mathrm{Na}_{2} \mathrm{O}$ como sexto componente baja a $1.280^{\circ} \mathrm{C}$. En general, todos los componentes menores que se disuelvan en la fase líquida reducirán la temperatura de su aparición.

Los mineralizadores en la interfase sólido-líquido (modificadores de la cinética de disolución de $\mathrm{C}_{2} \mathrm{~S}$ y $\mathrm{CaO}$ en la fase líquida) actúan aumentando la capacidad de "mojado" de la fase líquida, es decir, reduciendo la tensión superficial y, en consecuencia, permitiendo participar en el proceso de disolución una fracción más elevada de las superficies expuestas de las fases sólidas.

Los mineralizadores en fase líquida, por el contrario, aceleran la difusión iónica, reduciendo la viscosidad del líquido.

En la tabla II se muestra la composición del "líquidus", según Butt y Timashev (23), en el sistema cuaternario principal, a dos temperaturas $\left(1.338^{\circ} \mathrm{C}\right.$-aparición de fase líqui$\left.\mathrm{da}-\mathrm{y} 1.450^{\circ} \mathrm{C}\right)$.

TABLA II

\begin{tabular}{|c|c|c|}
\hline Componente & $\begin{array}{c}\text { Líquido 1 } \\
1.338^{\circ} \mathrm{C}\end{array}$ & $\begin{array}{c}\text { Líquido 2 } \\
1.450^{\circ} \mathrm{C}\end{array}$ \\
\hline $\mathrm{CaO}$ & 54,8 & 57,5 \\
$\mathrm{SiO}_{2}$ & 6,0 & 7,5 \\
$\mathrm{Al}_{2} \mathrm{O}_{3}$ & 22,7 & 22,6 \\
$\mathrm{Fe}_{2} \mathrm{O}_{3}$ & 16,5 & 12,9 \\
\hline $\mathrm{a}$ & 2,76 & 2,78 \\
$\mathrm{~b}$ & 0,016 & 0,025 \\
\hline
\end{tabular}

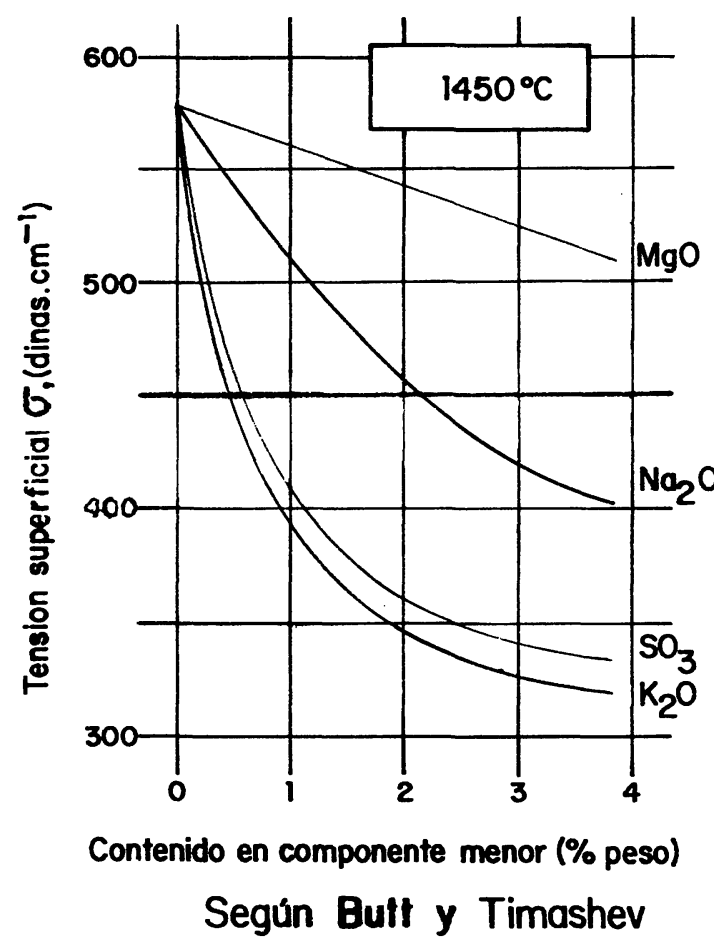

Fig. 3

Según dichos autores, para este sistema y en el intervalo $1.400-1.500^{\circ} \mathrm{C}$, es válida la siguiente fórmula que relaciona la tensión superficial con la viscosidad:

$$
\log \sigma=a-\frac{b}{\eta}
$$


en la que las constantes $a$ y $b$ son las de la tabla II, $\sigma$ la tensión superficial y $\eta$ la viscosidad. En los clínkeres normales, la tensión superficial entre $1.300-1.450^{\circ} \mathrm{C}$ es del orden de algunos cientos de dinas $\cdot \mathrm{cm}^{-1}$ y la viscosidad del orden del poise.

En la figura 3 se muestra la variación de la tensión superficial del líquido 2 a $1.450^{\circ} \mathrm{C}$ con adiciones a concentraciones variables de distintos componentes minoritarios.

En la figura 4 se presenta la variación de viscosidad, para el mismo líquido, la misma temperatura y los mismos componentes secundarios. Debe señalarse que los óxidos alcalinos, a igualdad de temperatura reducen la tensión superficial pero aumentan la viscosidad (iones no electronegativos de gran radio y baja carga).

Finalmente, existen mineralizadores en fase sólida que, a igualdad de temperatura, aumentan la disociación del $\mathrm{CaCO}_{3}$, mejoran la velocidad de los procesos de difusión en fase sólida y/o dan productos intermedios que favorecen o aceleran la formación de $\mathrm{C}_{3} \mathrm{~S}$, compuesto final en el proceso de creación del clínker.

A este tipo de sustancias pertenecen los cloruros alcalino y alcalinotérreos, los fluoruros, los sulfatos, los fosfatos, los nitratos, etc. Han dado resultados interesantes pero tienen otros aspectos desfavorables en el proceso industrial o no es rentable su aplicación. Por eso, la industria cementera sólo utiliza la fluorita y el yeso en casos muy particulares y con fines específicos.

La efectividad de algunos mineralizadores sobre la mezcla de los cuatro óxidos principales, en proporiciones normales en la práctica $(C / S=3 ; A / F=1,5$ y $A+f=10 \%)$, se muestra en la figura 5, donde puede observarse el efecto de $0,5 \%$ de $\mathrm{CaF}_{2}$ y $1 \%$ de adiciones de $\mathrm{MgO}$ y $\mathrm{SO}_{3}$ como $\mathrm{K}_{2} \mathrm{SO}_{4}$ sobre la aptitud a la cocción de un crudo (24).

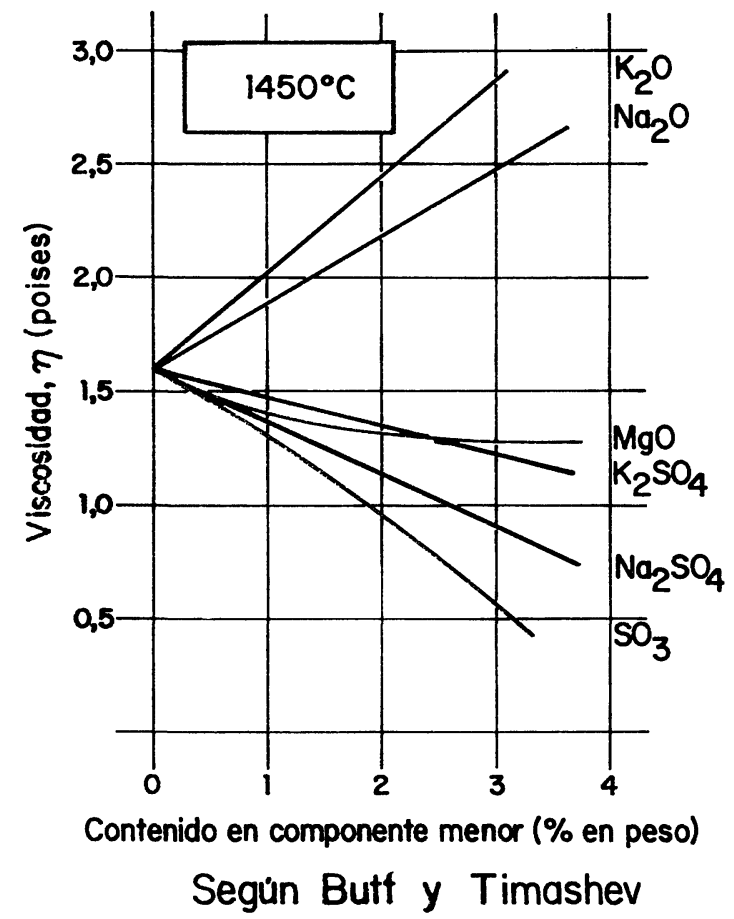

Fig. 4

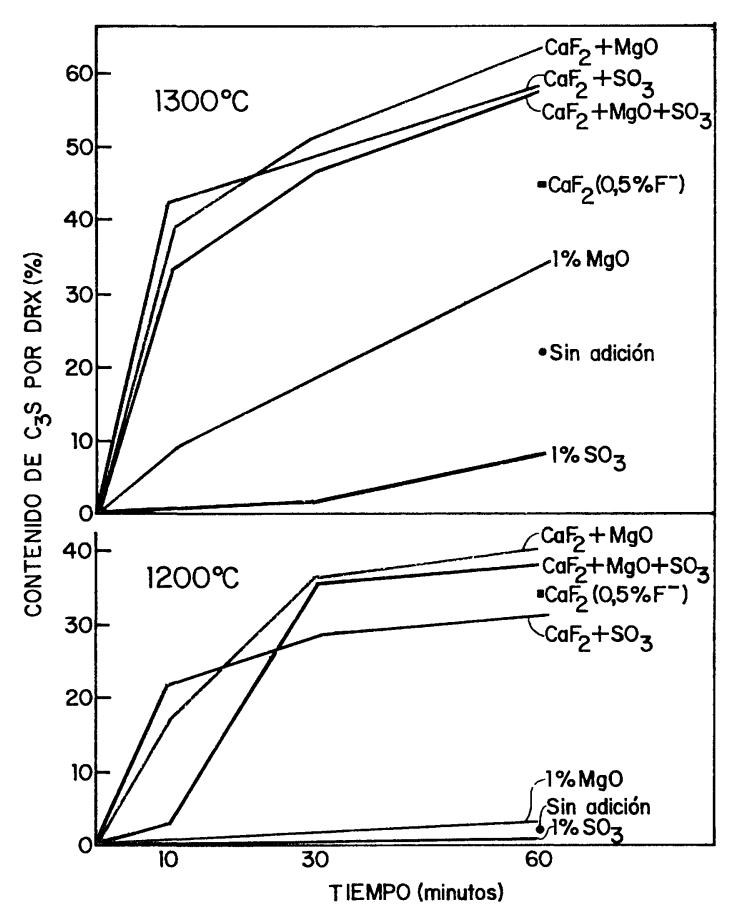

Fig. 5

Los componentes minoritarios, aparte de modificar las propiedades de la fase líquida, pueden trastocar el equilibrio de fases, formando nuevas soluciones sólidas e, incluso, nuevos compuestos. 
En algunos casos, estos componentes minoritarios, en concentración adecuada, modifican la estructura del clínker aumentando sus propiedades hidráulicas, bien por afectar las condiciones de formación y crecimiento de cristales (25) o bien por crear desordenes de redes por sustituciones isomórficas (23) (26).

\section{INTERES Y COMPORTAMIENTO PRACTICO DE ALGUNOS COMPONENTES MINORITARIOS}

A continuación se analizará brevemente la presencia, comportamiento e interés práctico de algunos componentes minoritarios, comenzando por los inevitables y siempre presentes en el proceso de clinkerización (álcalis, magnesio y sulfatos) y concluyendo con otros que, a veces, se incorporan buscando un fin específico.

\subsection{Alcalis}

Los álcalis se encuentran en minerales que acompañan siempre a los productos de la arcilla, tales como feldespatos, micas, etc. Los crudos europeos contienen, por término medio, de 0,5 a $2,0 \%$ de $\mathrm{K}_{2} \mathrm{O}$ y de 0,05 a $0,6 \%$ de $\mathrm{Na}_{2} \mathrm{O}$.

Por su volatilidad, los óxidos alcalinos dan lugar a ciclos en el interior del horno, incorporándose en parte en el producto cocido y siendo causa de posibles perturbaciones en el proceso normal de la cocción.

Por ello, aunque son fundentes, son los compuestos menos deseables desde el punto de vista tecnológico, procurando su eliminación del proceso, bien por separación de los polvos del electrofiltro (donde se acumulan en notable proporción), bien estableciendo un bypas en el intercambiador, o bien en el propio clínker en forma de sulfatos.

Estos sulfatos alcalinos en el clínker, influencian favorablemente la resistencia inicial de los cementos (hasta 3 días), pero la reducen a partir de 28 días (27).

\subsection{Magnesio}

Prácticamente, todas las calizas presentan magnesio en su composición en forma de carbonato o carbonato doble, tanto más cuanto más antigua es su formación. También puede acompañar a las arcillas como tal carbonato o como sustitución isomorfa en silicatos más o menos complejos.

Desde el punto de vista tecnológico, la presencia de magnesio facilita la reactividad de los crudos a altas temperaturas $\left(\sim 1.300^{\circ} \mathrm{C}\right)$ pero, desgraciadamente, su presencia en estado libre, a partir de ciertas proporciones que entran en soluciones sólidas con los minerales del clínker, es causa de inestabilidad de volumen de los cementos en la hidratación, razón por la cual limitan su contenido todas las normas del mundo.

\subsection{Sulfatos}

El azufre en forma de sulfatos, y rara vez en forma de sulfuros, puede encontrarse tanto en calizas como en margas o arcillas. A veces, se incorpora yeso o anhidrita al crudo como mineralizador o para modificar o equilibrar el circuito de álcalis.

El azufre y! los sulfuros, presentes tanto en el crudo como en el combustible, pasan normalmente a sulfatos en las condiciones del horno y combinan como tales. 
$\mathrm{El} \mathrm{CaSO}_{4}$ es un fundente y mineralizador eficaz, rebaja en más de $100^{\circ} \mathrm{C}$ la temperatura de aparición de la fase líquida y disminuye su viscosidad y tensión superficial (23).

Los sulfatos alcalinos son mineralizadores menos activos que el sulfato cálcico. Sin embargo, favorecen, lo mismo que los $\mathrm{Cl}^{1-}$ y los $\mathrm{F}^{1-}$, la formación de espurrita $\left(2 \mathrm{C}_{2} \mathrm{~S} \mathrm{CaCO}_{3}\right)$ estable hasta $1.298^{\circ} \mathrm{C}$, que es un "intermediario" para la formación rápida de $\mathrm{C}_{3} \mathrm{~S}$ (mineralizador en fase sólida).

Finalmente, los sulfatos juegan un papel muy importante en la formación de costras y anillos, fenómenos perturbadores en el proceso normal de cocción (28).

\subsection{Fluoruros}

El fluor está siempre presente en los crudos, aunque en pequeña proporción. A veces, se incorpora para mejorar su reactividad.

La presencia de fluoruros determina la formación de espurrita a $850^{\circ} \mathrm{C}$ que descompone formando $\mathrm{C}_{2} \mathrm{~S}$ y $\mathrm{CaO}$ en un estado especialmente activo para formar $\mathrm{C}_{3} \mathrm{~S}$. Por ello, la formación de éste comienza a $150-200^{\circ} \mathrm{C}$ menos que en los crudos sin fluoruros.

Con contenidos en $\mathrm{F}^{1-}$ superiores a $0,5-0,6 \%$ se reducen las resistencias mecánicas del producto final, por la presencia de $\mathrm{C}_{3} \mathrm{~S} . \mathrm{CaF}_{2}$, compuesto de baja hidraulicidad (29).

En la práctica como aportador de fluoruros se emplea generalmente la fluorita, por su costa más bien reducido y facilidad de manipulación. Su aplicación se ha extendido, no sólo a la fabricación ya tradicional de clínker de cemento blanco, sino también a los clínkeres normales, buscando ahorro de energía y un posible aumento de la capacidad de los hornos.

\subsection{Fosfatos}

El fósforo se encuentran en cantidades variables en rocas sedimentarias de origen orgánico, así como en muchos subproductos de la industria siderúrgica empleados frecuentemente en los crudos (escoria de horno alto, por ejemplo).

Se ha reconocido el efecto positivo de los fosfatos en todas las reacciones que tienen lugar en la cocción del clínker, tanto en fase sólida como en presencia de líquido (30).

En la práctica, la hidraulicidad de los clínkeres fosfáticos, muy estudiados por los investigadores de la Building Research Station de Garston (Inglaterra), muestra una ligera mejoría para bajos contenidos de $\mathrm{P}_{2} \mathrm{O}_{5}(0,5-1,0 \%)$ por la estructura particular de las soluciones sólidas que forma con el $\mathrm{C}_{3} \mathrm{~S}$. Por encima de estos límites baja considerablemente el poder hidráulico, al reducirse la proporción de $\mathrm{C}_{3} \mathrm{~S}$ y aumentar la proporción de los $\mathrm{C}_{2} \mathrm{~S} \alpha$ y $\alpha$, en detrimento de la forma $\beta$.

Desde el punto de vista de tecnología del proceso, los fosfatos reducen los ciclos de cloruros en el horno, evitando la formación de depósitos de espurrita.

\subsection{Compuestos de titanio}

El titanio se presenta generalmente en forma de ilmenita o rutilo, acompañando a arcillas, lateritas, basaltos, etc.

Es un buen fundente y mineralizador. En la práctica, $1 \%$ de $\mathrm{TiO}_{2}$ sobre clínker, es sufi- 
ciente para rebajar de 50 a $100^{\circ} \mathrm{C}$ la temperatura de aparición de la fase líquida (31). Además reduce la tensión superficial del líquido y provoca un descenso de la viscosidad proporcional a la concentración. Esto facilita la formación de alita con una eficacia superior, incluso, a la proporcionada por el $\mathrm{CaF}_{2}$.

Por su radio iónico, el $\mathrm{Ti}^{4+}$ puede sustituir al $\mathrm{Fe}^{3+}$ y al $\mathrm{Al}^{3+}$ en el ferroaluminato y aluminato cálcicos respectivamente; lo mismo ocurre con el $\mathrm{Si}^{4+}$ de los silicatos, singularmente del tricálcico. En la realidad el mineral con más tendencia a su enriquecimiento en titanio es el ferroaluminato, que llega a contener hasta $18 \%$ de $\mathrm{TiO}_{2}$ en peso.

Los clínkeres con un contenido en $\mathrm{TiO}_{2}$ del orden del $1 \%$ manifiestan resistencias, entre 3 y 90 días, hasta en un $20 \%$ superiores a las de un clínker normal (31).

\subsection{Compuestos de cromo}

El cromo en los cementos, lo mismo que el titanio, procede fundamentalmente del componente ácido del crudo, de las escorias siderúrgicas y del desgaste de placas y bolas en los molinos y, excepcionalmente, como resultado del desgaste del refractario.

Los compuestos de cromo reducen, a temperatura constante, la viscosidad de la fase líquida y, para concentraciones superiores a $2 \%$ de $\mathrm{Cr}_{2} \mathrm{O}_{3}$ en dicha fase, también reducen la tensión superficial.

Según parece, el cromo sustituye a la sílice de los silicatos formando soluciones sólidas, hasta un límite de $1,7 \%$ (expresado como $\mathrm{Cr}_{2} \mathrm{O}_{3}$ ) en el $\mathrm{C}_{3} \mathrm{~S}$ y $8,6 \%$ en el $\mathrm{C}_{2} \mathrm{~S}$, estabilizando su forma $\beta$.

En la práctica, las proporciones no deben pasar del $1 \%$ en peso, y en esta medida, se aumenta la resistencia inicial del clínker (25) debido, quizás, a un defecto de red de la solución sólida con el $\mathrm{C}_{3} \mathrm{~S}$. La presencia simultánea de fluor y manganeso favorece la acción del cromo.

\section{FINAL}

En el marco del ahorro energético en la industria del cemento, aparte de métodos de eficacia ya reconocida, debe contemplarse también la posible influencia de la composición quimicomineralógica de las materias primas.

Es cierto que el cemento ha de fabricarse donde hay posibilidades de mercado, debiendo emplearse los recursos materiales que yacen en un radio de acción económicamente rentable, pero, también es cierto que esto ocurre porque, hasta hace muy poco tiempo, se manejaban casi exclusivamente conceptos químicos con limitaciones, a veces muy estrechas, en cuanto a composición; hoy día, el abanico de posibilidades ha crecido al ampliarse el conocimiento del proceso de cocción y comprobarse experimentalmente el comportamiento de sistemas más complejos, con resultados francamente positivos en cuanto a calidad de producto y ahorro energético se refiere.

En los crudos convencionales, las diferencias en cuanto al consumo energético se refiere, el origen y naturaleza de las materias primas puede conducir a consumos que divergen en un $20 \%$ para la energía térmica y un $50 \%$ en la energía eléctrica, a igualdad de todo lo demás (32).

Hoy ya se habla de cementos que, empleando materias primas privilegiadas (lodos rojos 
desecho de la fabricación de alúmina, schlams de cenizas de lignitos pardos, etc.), pueden reducir el consumo de combustible de 20 a $25 \%$ en relación con las materias primas tradicionales - cementos de $\mathrm{C}_{2} \mathrm{~S}$ activo-

También se habla de los cementos de "alinita", posibles de clinkerizar a $1.000-1.100^{\circ} \mathrm{C}$, tratando las materias primas convencionales en presencia de $\mathrm{CaCl}_{2}$ como fundente-mineralizador.

Estas últimas líneas son las que marcan las investigaciones hoy día, con gran participación de los componentes menores: posibilidad de preparar tipos de alita estables a más baja temperatura o tipos de belita más activos hidráulicamente que los que hoy se fabrican.

\section{BIBLIOGRAFIA}

(1) G. Mussgnung (1953): “Comportamiento a la sinterización del crudo de cemento". Tagunsber. der Zementindustrie H. n. 7, 33, Zement-Kalk-Gips 6, 46.

(2) P. Longuet y B. Courtaul (1964): "Aptitud a la clinkerización de los crudos de fábricas de ceinento". Rev. Mat. Constr. n.० 585, 174.

(3) H. Kock, G. REY y F. BECKer (1974): "Un método estadístico para determinar la aptitud a la cocción de crudos de cemento". 6. Intern. Congr. Chem. Cement Moscú.

(4) U. LUdwig y G. RUCKensteiner (1974): "Sobre la aptitud a la cocción del crudo de cemento" Cement and Concrete Research, 4, 239.

(5) J. P. Sulikowkr (1968): “Aptitud a la cocción de crudos" Proc. 5. Intern. Symp. Chem. Cement Tokio P. I., 106.

(6) R. Blaise, M. Musikas y H. Tredrez (1971): "Nuevo método de determinación cinética de la aptitud a la cocción de un crudo de fábrica de cemento". Rev. Mat. Constr. n.* 674-75, 287.

(7) P. P. ZozurJa y E. A. Ropronov (1981): "Formación de minerales y capacidad reactiva de los crudos de cemento portland". 7.' Congr. Intern. Chim. Ciment, vol. II, I-201.

(8) E. Spohn, E. Woermann y D. KNöfel (1968): "Un perfeccionamiento de la fórmula del standard de cal". Proc. 5.॰ Intern. Symp. Chem. Cement, Tokyo, P. I., 172.

(9) N. H. Christensen (1979): “Aptitud a la cocción de crudos de cemento a 1.400C. I. El efecto de la composición química". Cement and Concrete Research, 9, 219.

(10) E. Fundal (1979): “La aptitud a la cocción de crudos de cemento". World Cement Technology 10, n.० 6, 195-6; 199-200; 203-204.

(11) B. Vosteen (1974): "Precalentamiento y calcinación total del crudo de cemento en un sistema en suspensión gaseosa". Zement-Kalk-Gips, 27, 443

(12) A. Müller, B. Dahm y J. Stark (1979): “Contribución a la cinética de la descomposición del carbonato cálcico". Zement-Kalk-Gips, 32, 78.

(13) W. L. DE KEYSER (1965): "Estudio sobre las reacciones en estado sólido entre $\mathrm{SiO}_{2}, \mathrm{Al}_{2} \mathrm{O}_{3}, \mathrm{MgO}$ $\mathrm{CaO}, \mathrm{Fe}_{2} \mathrm{O}_{3}, \mathrm{ZrO}_{2}$ ". Proc. 8..$^{\circ}$ Conf. Silicate Industr. Budapest, 133.

(14) N. H. Christensen y K. A. Simonsen (1970): "Difusión en el clínker de cemento portland" J. Amer. Cer. Soc. 53, 361 .

(15) N. H. Christensen (1978): "Los efectos de la magnesia en la combinación de la cal en el clínker". World Cement Techn. 9, 223.

(16) F. Soria (1963): “Contribución al estudio de los cementos portland resistentes a sulfatos". Monografía IETcc, n.o 230, Madrid.

(17) S. P. Makashev (1974): "Efecto de las propiedades fisicoquímicas del crudo sobre su reactividad y sobre el proceso de mineralogénesis del clínker". Proc. 6. Intern. Symp. Chem. Cement. Moscú.

(18) W. Goerg (1956): "Reacciones entre la cal y los minerales de la arcilla" Tonindustrie Zeitung, 80, 219.

(19) O. P. MChedlov-Petrosyan y otros (1975): "Métodos termodinámicos para estudiar la reactividad de crudos y cementos". Tsement, 41, n. $.^{\circ} 10,17$.

(20) J. Talaber (1968): "Difusión en fase sólida de CaO en sustancias minerales". Proc. 9. Conf. Silicate Ind. Budapest, 171. 
(21) J. A. WolfE (1955): “¿Qué considerar para seleccionar materias primas para cemento?”. Rock Prod. 58, agosto, 132.

(22) R. Bucchr (1981): "Aspectos sobre el papel de componentes menores en el clínker de cemento". World Cement Tech., junio, 210.

(23) Y. M. Butr, V. V. Tamashev y A. P. Osokin (1974): "El mecanismo de las procesos de formación del clínker y la modificación de su estructura". 6. Intern. Congr. Chem. Cement. Moscú.

(24) W. A. KLEEM y J. JAwED (1981): "Mineralizadores y fundentes en el proceso de clinkerización. III. Aptitud a la cocción de crudos sintéticos industriales". 7. Congr. Intern. Chim. Ciments, vol. II, I-151.

(25) W. KURDowskr (1974): "Influencia de componentes minoritarios sobre la actividad hidráulica del clínker de cemento portland". 6. Intern. Congr. Chem. Cement. Moscú.

(26) M. REgourd y A. GuINIER (1974): "La cristalquímica de los constituyentes del clínker de cemento portland". 6.' Intern. Congr. Chem. Cement. Moscú.

(27) J. Gebaugr y M. Kristmann (1979): "La influencia de la composición del clínker industrial sobre las propiedades del cemento $\mathrm{y}$ del hormigón". World Cement Techn. 10, 46.

(28) H. M. Sylla (1974): "Investigación sobre la formación de costras y anillos en el horno rotatorio de cemento". Zement-Kalk-Gips, 27, 449.

(29) J. H. WELCH y W. GUTT (1960): "El efecto de componentes menores sobre la hidraulicidad de los silicatos cálcicos". Proc. 4. Intern. Symp. Chem. Cement. Washington 1, 59.

(30) K. Akatsu, K. MaEda e I. Ireda (1970): "Fil efecto de $\mathrm{Cr}_{2} \mathrm{O}_{3}$ y $\mathrm{P}_{2} \mathrm{O}_{5}$ sobre la resistencia y color del clínker de cemento portand". Rev. 24* Gen. Meet. Cem. Assoc. Japan. Tokyo, 20.

(31) D. KNöFrL (1977): "Influencia del $\mathrm{TiO}_{2}$ en las propiedades de los clínkeres de cemento portland y del cemento portland". Zement-Kalk-Gips, 30, 191.

(32) R. G. Gouda (1981): "Ffecto de las materias primas sobre el proceso de fabricación y propiedades del cemento". 7.' Congr. Intern. Chim. Ciment, París, bol. II, I-235.

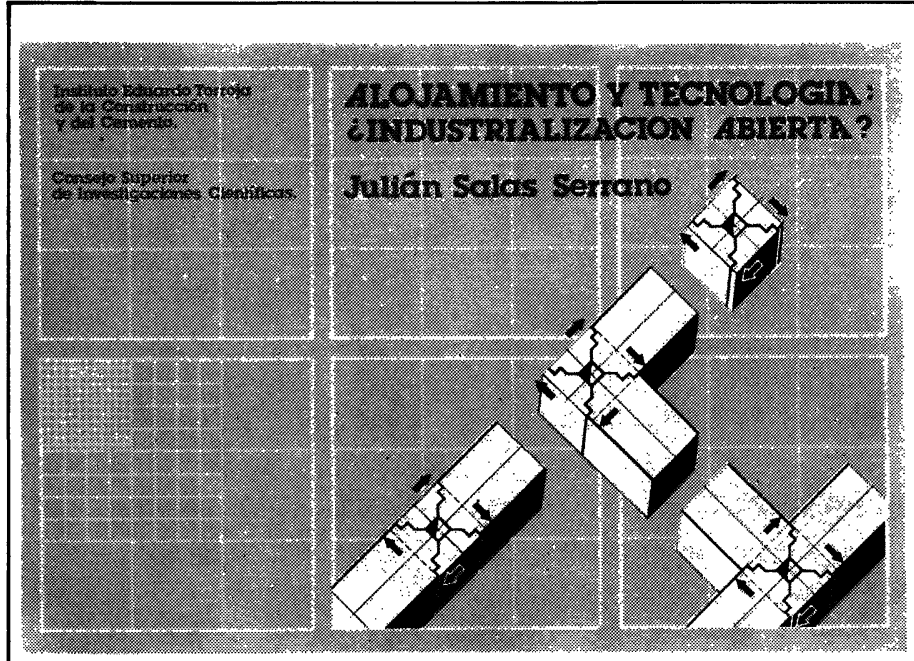

\section{ALOJAMIENTO Y TECNOLOGIA: ¿INDUSTRIALIZACION ABIERTA?}

JULIAN SALAS, ING. IND. (I.E.T.c.C.)

Un volumen de 160 páginas, 109 figuras y 16 tablas. Ta-

maño $240 \times 168 \mathrm{~mm}$. Encuadernado en rústica. Precios:

España, 1.200 ptas; extranjero, 17 \$ USA.

\section{SUMARIO:}

Prólogo Prof. G. Ciribini.

\section{Introducción}

Capítulo 1. - La industrialización en las proclamas y manifiestos de arquitectura.

Capítulo 2.-¿Réquiem por la construcción industrializada?

Capitulo 3.-Algunos conceptos básicos.

Capitulo 4. ¿ ¿Proyecto tradicional, construcción industrializada?

Capitulo 5.-Componentes.

Capitulo 6. - La coordinación dimensional hoy.

Capítulo 7.-Flexibilidad, intercambiabilidad y catálogos. Capitulo 8. - Industrialización, normativa y calidad.

Capitulo 9.-Reflexiones finales.

publicación del

INSTITUTO EDUARDO TORROJA 